Results Mean NMT warm-up exercise test scores were $72 \%$ (SD: $13 \%$ ) for the control and $71 \%$ (SD: 13\%) for the intervention workshop. Mean change in NMT warm-up self-efficacy scores were 0.98 (SD: 1.33) for the control and 1.77 (SD: 1.19) for the intervention workshop. Multivariable linear regression analyses indicated that workshop delivery method was not associated with the exercise test score $(b=-3.45$, $95 \%$ CI: -10.80 to $3.91, \mathrm{R}^{2}=0.13$ ) but was associated with a greater difference in change of self-efficacy scores for the intervention workshop $(b=0.97,95 \%$ CI: 0.26 to 1.89 , $\mathrm{R}^{2}=0.13$ ).

Conclusions A P2P learning technology integrated instructional workshop did not differentially impact coaches' ability to identify exercise errors, but it did increase coaches' selfefficacy in identifying exercise errors compared to a standard workshop.

\section{BUMBLED VIBRATION: 144 HZ VIBRATION DOES NOT ENHANCE SHOULDER CONTROL}

Amber Winton, Nicholas Andriani, Erica Marshall, James Lynch. Florida Southern College, Lakeland, FL, USA

\subsection{6/bjsports-2021-IOC.403}

Background Stability of the upper extremity is critical for injury prevention. Vibration may improve proprioception, reducing injury risk. However, traditional vibration methods may be expensive and require a significant level of skill by the technician.

Objective Our purpose was to investigate whether an acute bout of shoulder exercise performed with an inexpensive, user-friendly vibration toy ball (BumbleBall, Cardinal Laboratories) improves shoulder position sense and joint control.

Design Participants completed an acute bout of shoulder exercise with and without vibration on separate visits. Prior to the exercise bout, participants were assessed for motion sway (path length) using a custom iPhone application previously shown reliable. Path length was reassessed following the exercise bout. A 2-way repeated measures ANOVA was used to determine differences between conditions (vibration/no vibration) and time (pre/post). Significant main effects were examined using paired t-tests $-\mathrm{p}<0.05$.

Setting Liberal arts undergraduate institution.

Patients (or Participants) Thirty subjects (age 18-22) completed all trials. Subject level of activity ranged from non-athletes to collegiate athletes. Exclusion criterion was an upper extremity injury in the previous month.

Interventions (or Assessment of Risk Factors) Sessions started with an app measure. Each subject held the phone on the palm, arm forward at eye level for 20 seconds each arm for all trials. Each subject then completed a full can exercise set with the randomly assigned BumbleBall state (vibration/no vibration), and then repeated the app stability measure. The toy vibrates at $144 \mathrm{Hertz}$ with an amplitude of $8 \mathrm{~mm}$.

Main Outcome Measurements Average path length before and after use of the BumbleBall (vibration/no vibration) assessed this intervention.

Results There was a significant $\left(p=0.009\right.$ and $\left.\eta_{p}{ }^{2}=0.27\right)$ main effect of time. There were no significant $(p>0.05)$ 2-way interactions between condition and time.

Conclusions The vibration of this inexpensive toy at $144 \mathrm{~Hz}$ did not improve shoulder proprioception.

\section{EVALUATING EXERCISE FIDELITY DURING NEUROMUSCULAR TRAINING PROGRAMS USING WEARABLE TECHNOLOGY}

'Lauren Benson, ${ }^{1}$ Anu Räisänen, ${ }^{1}$ Sartaj Sidhu, 1,2,3,4,5Carolyn Emery. ${ }^{1}$ Sport Injury Prevention Research Centre, Faculty of Kinesiology, University of Calgary, Calgary, Canada; ${ }^{2}$ McCaig Bone and Joint Institute, Cumming School of Medicine, University of Calgary, Calgary, Canada; ${ }^{3}$ Alberta Children's Hospital Research Institute, University of Calgary, Calgary, Canada; ${ }^{4}$ Department of Community Health Sciences, Cumming School of Medicine, University of Calgary, Calgary, Canada; ${ }^{5}$ Department of Pediatrics, Cumming School of Medicine, University of Calgary, Calgary, Canada

\subsection{6/bjsports-2021-IOC.404}

Background Evaluating exercise fidelity during neuromuscular training (NMT) warm-ups (i.e., completing prescribed repetitions and performing exercises correctly) is important to inform the dose-response relationship of warm-up programs. Wearable technology can be used to measure exercise fidelity. Objective To determine the accuracy of measuring NMT exercise volume and quality with wearable technology.

Design Cross-sectional study

Setting Youth basketball; Calgary, Canada

Participants Twenty-seven youth basketball players

Assessment of Risk Factors Players wore a triaxial accelerometer on the lower back during an NMT warm-up with concurrent video recording. A trained observer (physiotherapist) used an observation tool to determine whether each athlete performed the prescribed exercise volume and rate posture.

Main Outcome Measurements The number of repetitions during running, skipping and jumping were extracted from the accelerometer signal using a custom peak detection algorithm and compared to the prescribed exercise volume. The algorithm accuracy was calculated as a percentage, with the trained observer evaluation through video-analysis considered the gold standard.

For the plank, participants were evaluated on 'Good Posture (straight body, head to ankle)' and received a score of 'Yes,' 'No,' or 'Partial.' The coefficient of variation (CV) of the accelerometer signal in all three axes was compared for the three fidelity assessment outcomes.

Results The algorithm had an accuracy of $100 \%$ for the running, skipping and jumping exercise volume.

Participants who scored 'Yes' had a lower CV in the medial-lateral (median: 47.2\%) and vertical (42.3\%) axes, than participants who scored 'Partial' (85.4\% and 67.6\%) and 'No' (115.1\% and $115.5 \%)$. There were no differences in $\mathrm{CV}$ in the anterior-posterior axis.

Conclusions A custom algorithm can be used to measure the number of running, skipping and jumping repetitions. The variability of the accelerometer signal can identify postural changes during a plank. Accelerometer signals may be used to evaluate movement quantity and quality during NMT.

\section{LONGITUDINAL CHANGES IN FORCE PLATE MEASURES ARE VALID INDICATORS OF MUSCULOSKELETAL HEALTH IN PROFESSIONAL AMERICAN FOOTBALL PLAYERS}

${ }^{1,2}$ Timothy Hewett, ${ }^{3}$ Kate Webster, ${ }^{4}$ Sarah Cohen, ${ }^{4}$ Phillip Wagner. ${ }^{1}$ Hewett Global Consulting, Minneapolis, USA; ${ }^{2}$ Rocky Mountain Consortium for Sports Medicine Research, Edwards, USA; ${ }^{3}$ La Trobe University, Melbourne, Australia; ${ }^{4}$ Sparta Science, Menlo Park USA

10.1136/bjsports-2021-IOC.405 